\title{
Statistical properties of spontaneous synchrotron radiation with arbitrary degree of coherence
}

\author{
Ihar Lobach $\odot^{*}$ \\ The University of Chicago, Department of Physics, Chicago, Illinois 60637, USA \\ Valeri Lebedev®, Sergei Nagaitsev®, ${ }^{\dagger}$ Aleksandr Romanov, \\ Giulio Stancari®, and Alexander Valishev \\ Fermi National Accelerator Laboratory, Batavia, Illinois 60510, USA \\ Aliaksei Halavanau and Zhirong Huang \\ SLAC National Accelerator Laboratory, Stanford University, Menlo Park, California 94025, USA \\ Kwang-Je Kim ${ }^{\dagger}$ \\ Argonne National Accelerator Laboratory, Lemont, Illinois 60439, USA
}

(Received 20 December 2019; accepted 31 August 2020; published 11 September 2020)

\begin{abstract}
In a storage ring, turn-to-turn fluctuations in the intensity of spontaneous synchrotron radiation occur due to two mechanisms. The first mechanism is the quantum uncertainty in the number of emitted photons. The second mechanism is the turn-to-turn variations in the relative positions of classical pointlike electrons in the bunch. We present a unified description of both effects in the framework of quantum optics. We derive an equation for the fluctuations for an arbitrary degree of coherence, which generalizes previously reported results for temporally incoherent radiation. We compare the predictions of our calculation with a previous experiment at Brookhaven National Laboratory, where the latter mechanism was dominant and propose a new dedicated experiment in the Integrable Optics Test Accelerator (IOTA) at Fermilab, where the two mechanisms may have comparable contributions to the fluctuations. Finally, our calculation shows that the magnitude of the fluctuations is rather sensitive to the dimensions and the shape of the electron bunch, thereby indicating possible applications in beam instrumentation. In particular, the small vertical size of the flat beams in IOTA may be estimated via these fluctuations, whereas measurement by a conventional synchrotron radiation monitor is difficult due to the diffraction limit.
\end{abstract}

DOI: 10.1103/PhysRevAccelBeams.23.090703

\section{INTRODUCTION}

In the last few decades there were several experiments regarding the statistical properties of incoherent synchrotron radiation produced by electron bunches in storage rings and linear accelerators [1-5]. Pulse-to-pulse variations in the radiated energy were also studied theoretically. It was shown in Refs. [2,3] that in some cases the rms electron bunch length can be measured via these fluctuations. Moreover, Refs. [4,5] suggest that if the fluctuations in the radiation spectrum are measured with a high

\footnotetext{
*ilobach@uchicago.edu

Also at The Enrico Fermi Institute, The University of Chicago, Chicago, Illinois 60637, USA.

Published by the American Physical Society under the terms of the Creative Commons Attribution 4.0 International license. Further distribution of this work must maintain attribution to the author(s) and the published article's title, journal citation, and DOI.
}

resolution spectrometer, then even the shape of the electron bunch can be reconstructed. These observations, combined with the fact that fluctuations of the same nature are present in SASE FELs [6-11], make the study of fluctuations in synchrotron radiation relevant to better understanding of beam dynamics and, potentially, to beam diagnostics.

The number of photons, radiated by an electron bunch in an external electromagnetic field (undulator, wiggler, bending magnet, etc.), fluctuates from pass to pass due to the following two mechanisms [12]. The first mechanism is the photon shot noise, related to the quantum discrete nature of light. This effect would exist even if there was only one electron. Indeed, the electron would radiate photons with Poisson statistics [13-15]. The second mechanism is related to the interference of fields radiated by different electrons. Changes in relative electron positions and velocities inside the bunch result in fluctuations in intensity, and, consequently, in the number of photons. In a storage ring, the effect arises because of the betatron motion, synchrotron motion, radiation induced diffusion, 
etc.; in linacs, there are additionally bunch-to-bunch fluctuations in beam intensity.

For dense bunches, the fluctuations in the number of emitted photons are usually dominated by the latter mechanism [7], as was the case in Refs. [1-5]. In this paper, we present a unified description of both mechanisms in the framework of quantum optics. Below, we consider a single electron bunch circulating in a storage ring. The number of electrons in the bunch is constant. We consider the radiation generated in one of the synchrotron radiation sources in the ring, e.g., in an undulator, a wiggler, a bending magnet, etc. In Sec. II, we derive equations for the mean and the variance of the number of detected photons for an electron bunch of arbitrary shape. These equations apply to any degree of coherence of the radiation. Then, in Sec. III, the general equations are simplified for the case of temporally incoherent radiation, and an example of a Gaussian electron bunch is considered. Section IV discusses several cases, where simple closed-form expressions can be obtained. In Sec. V, our calculations are compared with the empirical data from the previous experiment carried out at Brookhaven National Laboratory [1]. Unfortunately, the experiment's parameters are not known to us with sufficient accuracy. Therefore, in Sec. VI, we propose a new experiment in the IOTA storage ring [16-18], where all relevant parameters will be measured with good accuracy.

\section{DERIVATION OF FLUCTUATIONS}

Let us assume that we have a detector that can measure the number of detected photons $\mathcal{N}$ during one revolution in a ring. The operator corresponding to this observable will be denoted by $\hat{\mathcal{N}}$. The operation principle of the detector will be discussed later. In this section, we want to calculate the average number of detected photons $\langle\mathcal{N}\rangle$ and its turnto-turn variance $\operatorname{var}(\mathcal{N})$ in the framework of quantum optics. We consider quantized electromagnetic field of the radiation. The electrons are classical pointlike particles with equal velocities before entering the synchrotron radiation source. Quantitatively, it means that the electron momentum spread $\sigma_{p}$ is sufficiently small, so that all of them produce radiation with approximately the same spectrum. Also, the beam divergence is negligible compared to the radiation divergence $[2,7,19]$,

$$
\sigma_{x^{\prime}}, \sigma_{y^{\prime}} \ll \sigma_{r^{\prime}}
$$

These conditions are satisfied in the experiments reported in Refs. [1,2,17], for example.

\section{A. Density operator formalism}

The density operator [14,20-22] for the radiation is

$$
\hat{\rho}=\int d \xi p(\xi)|\psi(\xi)\rangle\langle\psi(\xi)|,
$$

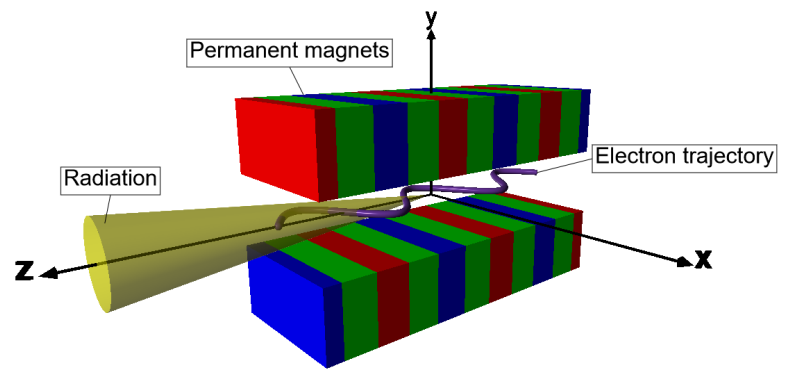

FIG. 1. Chosen coordinate system for the description of undulator (wiggler) radiation.

where $|\psi(\xi)\rangle$ represents the state of the radiation field after the passage of the electron bunch through the synchrotron radiation source. This state is a function of the initial relative positions of the electrons in the bunch. To signify this we use the symbol $\xi$,

$$
\begin{gathered}
\xi=\left(\boldsymbol{r}_{1}, \ldots \boldsymbol{r}_{n_{e}}\right), \\
d \xi=d \boldsymbol{r}_{1} \ldots d \boldsymbol{r}_{n_{e}},
\end{gathered}
$$

where $\boldsymbol{r}_{m}=\left(x_{m}, y_{m},-c t_{m}\right)$, here and below vector quantities will be represented by bold symbols; $n_{e}$ is the number of electrons in the bunch, $p(\xi)$ is the probability density function for the relative electron positions configuration $\xi$,

$$
\int d \xi p(\xi)=1
$$

In a more general case, $\xi$ should include the initial velocities of the electrons. Here they are assumed equal.

We choose a coordinate system where the $z$-axis is along the axis of the undulator (wiggler), and the $x$ - and $y$-axes are in the horizontal and vertical planes, respectively, as illustrated in Fig. 1. In a bending magnet, the z-axis can be chosen to point along the radiation traveling toward the detector.

We seek the expressions for the average number of detected photons $\langle\mathcal{N}\rangle$ and for the turn-to-turn variance of $\mathcal{N}, \operatorname{var}(\mathcal{N})$. In the density operator formalism [20],

$$
\begin{aligned}
\langle\mathcal{N}\rangle=\operatorname{Tr}(\hat{\rho} \hat{\mathcal{N}})=\int d \xi p(\xi)\langle\psi(\xi)|\hat{\mathcal{N}}| \psi(\xi)\rangle, \\
\operatorname{var}(\mathcal{N})=\operatorname{Tr}\left(\hat{\rho}(\hat{\mathcal{N}}-\langle\mathcal{N}\rangle)^{2}\right)=\operatorname{Tr}\left(\hat{\rho} \hat{\mathcal{N}}^{2}\right)-\langle\mathcal{N}\rangle^{2} \\
=\int d \xi p(\xi)\left\langle\psi(\xi)\left|\hat{\mathcal{N}}^{2}\right| \psi(\xi)\right\rangle-\langle\mathcal{N}\rangle^{2}
\end{aligned}
$$

We consider the regime of negligible electron recoil, and, thereby, classical electrons $[14,15,15]$. This is satisfied in all present storage rings. In this case, according to Ref. [14], the radiation is in a coherent state. We begin by considering the radiation in a cube with a finite side length $L$ and 
periodic boundary conditions. Hence, there are discrete optical modes with wave vectors $\boldsymbol{k}=(m, n, l) \times 2 \pi / L$, where $m, n, l$ are integers, not equal to zero simultaneously. For each wave vector $\boldsymbol{k}$, there are two perpendicular polarizations $\boldsymbol{e}_{s}(\boldsymbol{k}), s=1$, 2. For simplicity, we will consider only one polarization $s$. In this case, each mode is fully described by its wave vector $\boldsymbol{k}$. Therefore, instead of carrying the indices $m, n, l$, we will use a single symbol $\boldsymbol{k}$ as an index in a sum (or product) over all optical modes. Similarly, $\boldsymbol{k}$ will be used as a subscript of several functions, quantities, quantum states and operators to indicate which mode they pertain to. In this convention, the coherent state of the radiation field, as a direct product over all the optical modes, is

$$
|\psi(\xi)\rangle=\prod_{k}\left|\alpha_{k}(\xi)\right\rangle_{k}
$$

with

$$
\left|\alpha_{k}(\xi)\right\rangle_{k}=e^{-\frac{1}{2}\left|\alpha_{k}(\xi)\right|^{2}} \sum_{n_{k}} \frac{\alpha_{k}^{n_{k}}(\xi)}{\sqrt{n_{k} !}}\left|n_{k}\right\rangle_{k},
$$

where $\left|n_{k}\right\rangle_{k}$ represents the number state (Fock state) of the optical mode with wave-vector $\boldsymbol{k}$. According to Ref. [14],

$$
\alpha_{k}(\xi)=\frac{i}{\sqrt{2 L^{3} \hbar \omega}} \int d t d \boldsymbol{r} \boldsymbol{e}_{s}(\boldsymbol{k}) \cdot \boldsymbol{j}(\xi, \boldsymbol{r}, t) e^{i \boldsymbol{k} \cdot \boldsymbol{r}-i \omega t},
$$

where $\boldsymbol{j}(\xi, \boldsymbol{r}, t)$ represents the current density of the electron bunch with the initial relative arrangement $\xi, \hbar$ is the reduced Planck constant, $\omega=k c$, where $c$ is the speed of light.

The coherent state of each optical mode is the eigenstate of the annihilation operator corresponding to this mode,

$$
\hat{a}_{k}\left|\alpha_{k}(\xi)\right\rangle_{k}=\alpha_{k}(\xi)\left|\alpha_{k}(\xi)\right\rangle_{k}
$$

We postpone the calculation of $\alpha_{k}(\xi)$ until Sec. II C. Discussion of the regime, where both polarization components are registered, will be provided after Eq. (34).

\section{B. Detection operator}

The specifics of operation of the chosen light detector determine the form of the operator $\hat{\mathcal{N}}$. This operator has different forms for photodiodes, homodyne detectors, avalanche photodiodes and multiplexed detection schemes with many avalanche photodiodes [20]. In this paper, for the $\boldsymbol{k}$-dependent (and polarization dependent) quantum efficiency of a nonideal detector, $\eta_{k}$, we consider the model of a beam splitter, followed by an ideal detector, see [21] and Fig. 2.

The input-output relations for the beam splitter take the form

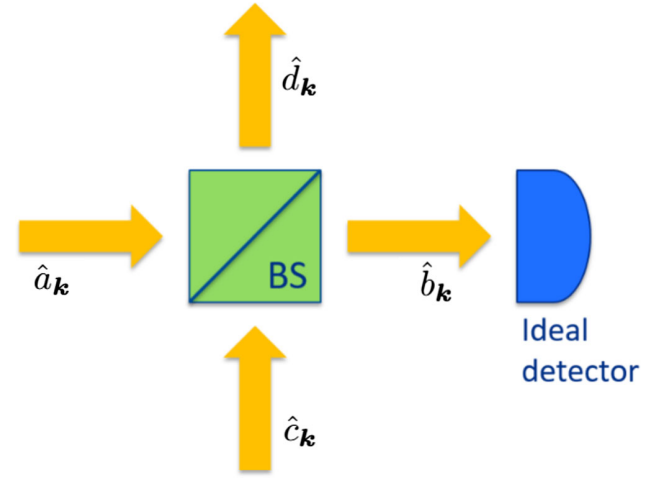

FIG. 2. The beam splitter model for quantum efficiency of a nonideal detector.

$$
\begin{aligned}
& \hat{b}_{k}=\sqrt{\eta_{k}} \hat{a}_{k}+i \sqrt{1-\eta_{k}} \hat{c}_{k}, \\
& \hat{d}_{k}=\sqrt{1-\eta_{k}} \hat{c}_{k}+i \sqrt{\eta_{k}} \hat{a}_{k},
\end{aligned}
$$

where $\hat{a}_{k}$ represents the incoming field, $\hat{c}_{\boldsymbol{k}}$ corresponds to the second input port, which is in the vacuum state in this model, $\hat{b}_{k}$ and $\hat{d}_{k}$ are the transmitted and reflected fields, respectively. This model is appropriate for quantum efficiency of a photodiode [20], where the number of detected photons $\mathcal{N}$ is determined as the number of photoelectrons coming from the photodiode. In addition, this model can take into account the spectral transmission of optical filters, lenses, reflectance of mirrors, detector acceptance.

Further, we assume that the operator for the total number of detected photons $\hat{\mathcal{N}}$ is a superposition over all modes. Then,

$$
\hat{\mathcal{N}}=\sum_{k} \hat{b}_{k}^{\dagger} \hat{b}_{k}
$$

In this model of the detector, the operator $\hat{\mathcal{N}}$ belongs to the Hilbert space composed of the Hilbert subspaces of both input ports of the beam splitter. Therefore, the vector state of the radiation $|\psi(\xi)\rangle$ has to be supplemented by the vacuum state of the second input port,

$$
|\psi(\xi)\rangle \rightarrow|0\rangle_{c}|\psi(\xi)\rangle
$$

where the subscript $c$ is used to indicate the Hilbert subspace of the second input port. When the substitution, Eq. (15), is carried out in Eqs. (6) and (7), the expectation values in these equations will take the following form,

$$
\begin{aligned}
\langle\psi(\xi)|\hat{\mathcal{N}}| \psi(\xi)\rangle & \rightarrow\left\langle\left.\psi(\xi)\right|_{c}\langle 0|\hat{\mathcal{N}}| 0\rangle_{c} \mid \psi(\xi)\right\rangle, \\
\left\langle\psi(\xi)\left|\hat{\mathcal{N}}^{2}\right| \psi(\xi)\right\rangle & \rightarrow\left\langle\left.\psi(\xi)\right|_{c}\left\langle 0\left|\hat{\mathcal{N}}^{2}\right| 0\right\rangle_{c} \mid \psi(\xi)\right\rangle .
\end{aligned}
$$

We calculate the expectation values in Eqs. (16) and (17) for the vacuum state of the second input port in the 
Appendix A. The results are as follows,

$$
\begin{gathered}
{ }_{c}\langle 0|\hat{\mathcal{N}}| 0\rangle_{c}=\sum_{k} \eta_{k} \hat{a}_{k}^{\dagger} \hat{a}_{\boldsymbol{k}}, \\
{ }_{c}\left\langle 0\left|\hat{\mathcal{N}}^{2}\right| 0\right\rangle_{c}=\sum_{\boldsymbol{k}} \eta_{\boldsymbol{k}} \hat{a}_{\boldsymbol{k}}^{\dagger} \hat{a}_{\boldsymbol{k}}+\sum_{\boldsymbol{k}_{1}, \boldsymbol{k}_{2}} \eta_{\boldsymbol{k}_{1}} \eta_{\boldsymbol{k}_{2}} \hat{a}_{\boldsymbol{k}_{1}}^{\dagger} \hat{a}_{\boldsymbol{k}_{2}}^{\dagger} \hat{a}_{\boldsymbol{k}_{2}} \hat{a}_{\boldsymbol{k}_{1}},
\end{gathered}
$$

these expressions are not scalars, they are operators in the Hilbert subspace of the first input port, to which the vector state $|\psi(\xi)\rangle$ belongs.

\section{General expressions for the mean and the variance of the number of detected photons}

The expectation values of the operators obtained in Eqs. (18) and (19) for the vector state $|\psi(\xi)\rangle$, defined in Eq. (8), can be calculated by using Eq. (11). When the results are used in Eqs. (6) and (7), the expressions for $\langle\mathcal{N}\rangle$ and $\operatorname{var}(\mathcal{N})$ in the beam splitter model of the detector take the following form,

$$
\langle\mathcal{N}\rangle=\sum_{k} \int d \xi p(\xi) \eta_{k}\left|\alpha_{k}(\xi)\right|^{2},
$$

$\operatorname{var}(\mathcal{N})=\langle\mathcal{N}\rangle+\int d \xi p(\xi)\left(\sum_{k} \eta_{k}\left|\alpha_{k}(\xi)\right|^{2}\right)^{2}-\langle\mathcal{N}\rangle^{2}$

Assuming a monoenergetic electron bunch with negligible beam divergence, see Eq. (1), we derived the following relation in Appendix B,

$$
\alpha_{k}(\xi)=\alpha_{k}^{(1)} \times \sum_{m} e^{i \tilde{k} \cdot r_{m}}
$$

where $\tilde{\boldsymbol{k}}=\left(k_{x}, k_{y}, k\right)$, noting that the $z$-component is $k=|\boldsymbol{k}|=\omega / c$, not $k_{z} ; \boldsymbol{r}_{m}=\left(x_{m}, y_{m},-c t_{m}\right)$ is the initial position of the $m$ th electron; $\alpha_{k}^{(1)}$ is calculated only for the reference electron, which can be chosen to be $m=1$ without loss of generality,

$$
\alpha_{k}^{(1)}=\frac{i e}{\sqrt{2 L^{3} \hbar \omega}} \int d t \boldsymbol{e}_{s}(\boldsymbol{k}) \cdot \boldsymbol{v}_{1}(t) e^{i \boldsymbol{k} \cdot r_{1}(t)-i \omega t},
$$

where $e$ is the electron charge, $\boldsymbol{r}_{1}(t)$ and $\boldsymbol{v}_{1}(t)$ are the trajectory and the velocity (as a function of time) of the reference electron in the synchrotron radiation source.

At this point, we can take the limit $L \rightarrow \infty$ and transition from discrete to continuous optical modes, i.e.,

$$
\left|\alpha_{k}^{(1)}\right|^{2} \rightarrow I_{k}^{(1)} d k
$$

where

$$
I_{\boldsymbol{k}}^{(1)}=\frac{e^{2}}{2(2 \pi)^{3} \hbar \omega}\left|\int d t \boldsymbol{e}_{s}(\boldsymbol{k}) \cdot \boldsymbol{v}_{1}(t) e^{i \boldsymbol{k} \cdot \boldsymbol{r}_{1}(t)-i \omega t}\right|^{2}
$$

Then, Eqs. (20) and (21) become, respectively,

$$
\begin{aligned}
\langle\mathcal{N}\rangle & =\int d \boldsymbol{k} \eta_{\boldsymbol{k}} I_{\boldsymbol{k}}^{(1)} \int d \xi p(\xi) \mathcal{J}_{\boldsymbol{k}}(\xi), \\
\operatorname{var}(\mathcal{N})= & \langle\mathcal{N}\rangle+\int d \boldsymbol{k}_{1} d \boldsymbol{k}_{2} \eta_{\boldsymbol{k}_{1}} I_{\boldsymbol{k}_{1}}^{(1)} \eta_{\boldsymbol{k}_{2}} I_{\boldsymbol{k}_{2}}^{(1)} \\
& \times \int d \xi p(\xi) \mathcal{J}_{\boldsymbol{k}_{1}}(\xi) \mathcal{J}_{\boldsymbol{k}_{2}}(\xi)-\langle\mathcal{N}\rangle^{2},
\end{aligned}
$$

where we introduced

$$
\mathcal{J}_{k}(\xi) \equiv\left|\sum_{m} e^{i \tilde{k} \cdot r_{m}}\right|^{2}
$$

Further, we consider an electron bunch, where the positions of the electrons are independent random variables with identical probability functions, i.e.,

$$
p(\xi)=p\left(\boldsymbol{r}_{1}\right) \ldots p\left(\boldsymbol{r}_{n_{e}}\right) .
$$

$\mathcal{J}_{k}(\xi)$ can be represented in the following form,

$$
\begin{aligned}
\mathcal{J}_{\boldsymbol{k}}(\xi) & =\sum_{m} e^{i \tilde{\boldsymbol{k}} \cdot \boldsymbol{r}_{m}} \sum_{n} e^{-i \tilde{\boldsymbol{k}} \cdot \boldsymbol{r}_{n}}=\sum_{m, n} e^{i \tilde{\boldsymbol{k}} \cdot\left(\boldsymbol{r}_{m}-\boldsymbol{r}_{n}\right)} \\
& =\sum_{m} 1+\sum_{m \neq n} e^{i \tilde{\boldsymbol{k}} \cdot\left(\boldsymbol{r}_{m}-\boldsymbol{r}_{n}\right)} \\
& =n_{e}+\sum_{m \neq n} e^{i \tilde{\boldsymbol{k}} \cdot\left(\boldsymbol{r}_{m}-\boldsymbol{r}_{n}\right)} .
\end{aligned}
$$

Therefore, the integral over $\xi$ in Eq. (26) can be calculated as

$$
\begin{aligned}
\int d \xi p(\xi) \mathcal{J}_{\boldsymbol{k}}(\xi)= & n_{e}+\int d \xi p(\xi) \sum_{m \neq n} e^{i \tilde{\boldsymbol{k}} \cdot\left(\boldsymbol{r}_{m}-\boldsymbol{r}_{n}\right)} \\
= & n_{e}+\sum_{m \neq n} \int p\left(\boldsymbol{r}_{m}\right) e^{i \tilde{\boldsymbol{k}} \cdot \boldsymbol{r}_{m}} d \boldsymbol{r}_{m} \\
& \times \int p\left(\boldsymbol{r}_{n}\right) e^{-i \tilde{\boldsymbol{k}} \cdot \boldsymbol{r}_{n}} d \boldsymbol{r}_{n} \\
= & n_{e}+n_{e}\left(n_{e}-1\right)|\mathcal{P}(\tilde{\boldsymbol{k}})|^{2}
\end{aligned}
$$

where the multiplier $n_{e}\left(n_{e}-1\right)$ comes from the sum over $m$ and $n$ from 1 to $n_{e}$, ignoring the diagonal terms, where $m=n$. Also, we defined the following function,

$$
\mathcal{P}(\tilde{\boldsymbol{k}}) \equiv \int p(\boldsymbol{r}) e^{i \tilde{k} \cdot \boldsymbol{r}} d \boldsymbol{r}
$$

The calculation of the integral over $\xi$ in Eq. (27) is lengthy. The details are provided in Appendix C. The final expressions for $\langle\mathcal{N}\rangle$ and $\operatorname{var}(\mathcal{N})$ are the following, 


$$
\begin{gathered}
\langle\mathcal{N}\rangle=n_{e} \int d \boldsymbol{k} \eta_{\boldsymbol{k}} I_{\boldsymbol{k}}^{(1)}\left(1+\left(n_{e}-1\right)|\mathcal{P}(\tilde{\boldsymbol{k}})|^{2}\right) \\
\operatorname{var}(\mathcal{N})=\langle\mathcal{N}\rangle+n_{e}\left(n_{e}-1\right) \int d \boldsymbol{k}_{1} d \boldsymbol{k}_{2} \eta_{\boldsymbol{k}_{1}} I_{\boldsymbol{k}_{1}}^{(1)} \eta_{\boldsymbol{k}_{2}} I_{\boldsymbol{k}_{2}}^{(1)}\left[\left|\mathcal{P}\left(\tilde{\boldsymbol{k}}_{1}-\tilde{\boldsymbol{k}}_{2}\right)\right|^{2}-\left|\mathcal{P}\left(\tilde{\boldsymbol{k}}_{1}\right) \mathcal{P}\left(\tilde{\boldsymbol{k}}_{2}\right)\right|^{2}\right. \\
\left.+\left(n_{e}-2\right)\left[\mathcal{P}^{*}\left(\tilde{\boldsymbol{k}}_{1}\right) \mathcal{P}\left(\tilde{\boldsymbol{k}}_{2}\right)\left(\mathcal{P}\left(\tilde{\boldsymbol{k}}_{1}-\tilde{\boldsymbol{k}}_{2}\right)-\mathcal{P}\left(\tilde{\boldsymbol{k}}_{1}\right) \mathcal{P}^{*}\left(\tilde{\boldsymbol{k}}_{2}\right)\right)+\text { c.c. }\right]\right] .
\end{gathered}
$$

Equations (33) and (34) are the most general results of this paper. They apply to any degree of coherence of the radiation (both transverse and temporal), for any number of the electrons in the bunch $n_{e}$, and for any probability function of each electron $p(\boldsymbol{r})$. If both polarization components are registered by the detector, the following substitution should be performed,

$$
\eta_{\boldsymbol{k}} I_{k}^{(1)} \rightarrow \sum_{s=1,2} \eta_{k, s} I_{k, s}^{(1)}
$$

In Eq. (33), the first term represents incoherent radiation. The second term constitutes coherent synchrotron radiation (CSR). The limit of fully coherent radiation can be obtained by assuming that all the electrons are concentrated in a single point. Using the Dirac delta function, $p(\boldsymbol{r})=\delta(\boldsymbol{r})$. In this limit, for any $\tilde{\boldsymbol{k}}$,

$$
\mathcal{P}(\tilde{\boldsymbol{k}})=1,
$$

and in Eq. (33) we obtain the expected result, that the intensity scales as $n_{e}^{2}$.

In Eq. (34), the first term, namely, $\langle\mathcal{N}\rangle$, is the photon shot noise contribution. It exists even in the case of a single electron, $n_{e}=1$. The second term arises from the variations in the relative positions of the classical pointlike electrons in the bunch. It vanishes in the case of fully coherent radiation, as one can see by inserting Eq. (36) in Eq. (34). However, it is non-zero in the case of incoherent or partially coherent radiation. Therefore, henceforth this term will be called the incoherence contribution to the fluctuations.

Further, we will consider possible simplifications of Eqs. (33) and (34) in specific cases. The form of Eqs. (33) and (34) for a Gaussian electron bunch is provided in Appendix D.

\section{REGIME OF TEMPORAL INCOHERENCE}

In this section, we will consider the regime of temporally incoherent radiation,

$$
\omega \sigma_{t} \gg 1,
$$

where $\sigma_{t}$ is the rms bunch duration. We will show that Eq. (34) agrees with the results obtained in [2], where this less general regime was studied classically.

Given Eq. (37), the integrals of the form of Eq. (32) with the arguments $\tilde{\boldsymbol{k}}_{1}$ and $\tilde{\boldsymbol{k}}_{2}$ will be negligible due to the fast oscillations of the phase factor. Only the integral with the argument $\tilde{\boldsymbol{k}}_{1}-\tilde{\boldsymbol{k}}_{2}$ will result in a nonvanishing contribution. Therefore,

$$
\langle\mathcal{N}\rangle=n_{e} \int d \boldsymbol{k} \eta_{k} I_{k}^{(1)}
$$

$$
\begin{aligned}
\operatorname{var}(\mathcal{N})= & \langle\mathcal{N}\rangle+n_{e}\left(n_{e}-1\right) \\
& \times \int d \boldsymbol{k}_{1} d \boldsymbol{k}_{2} \eta_{\boldsymbol{k}_{1}} I_{\boldsymbol{k}_{1}}^{(1)} \eta_{\boldsymbol{k}_{2}} I_{\boldsymbol{k}_{2}}^{(1)}\left|\mathcal{P}\left(\tilde{\boldsymbol{k}}_{1}-\tilde{\boldsymbol{k}}_{2}\right)\right|^{2} .
\end{aligned}
$$

Following the notation of [1,2] and [[7] p. 28], Eq. (39) can be re-written as

$$
\operatorname{var}(\mathcal{N})=\langle\mathcal{N}\rangle+\frac{1}{M}\langle\mathcal{N}\rangle^{2},
$$

where the parameter $M$ was introduced. In this paper it is defined as

$$
\frac{1}{M} \equiv\left(1-1 / n_{e}\right) \frac{\int d \boldsymbol{k}_{1} d \boldsymbol{k}_{2} \eta_{\boldsymbol{k}_{1}} I_{\boldsymbol{k}_{1}}^{(1)} \eta_{\boldsymbol{k}_{2}} I_{\boldsymbol{k}_{2}}^{(1)}\left|\mathcal{P}\left(\tilde{\boldsymbol{k}}_{1}-\tilde{\boldsymbol{k}}_{2}\right)\right|^{2}}{\left(\int d \boldsymbol{k} \eta_{\boldsymbol{k}} I_{\boldsymbol{k}}^{(1)}\right)^{2}},
$$

however, it can be identified with the number of coherent modes, defined in $[7,19,23]$, therefore we will use this name for the parameter $M$ from now on. Equation (41) is in agreement with Eq. (14) of Ref. [2], which was derived for a relative fluctuation of classical radiation intensity. Also, it agrees with a simple order-of-magnitude estimate of $M$, provided in Appendix F.

Equation (37) is satisfied in the Brookhaven experiment [1] and in the proposed experiment in IOTA [17,18], which will be discussed in Secs. V and VI, respectively. In both cases, $\omega \sigma_{t} \sim 10^{6}$.

There is one more possible simplification, which can be used for the above mentioned experiments. If we assume that the probability function can be decoupled into temporal and transverse components,

$$
p(\boldsymbol{r})=p_{t}(t) p_{\perp}\left(\boldsymbol{r}_{\perp}\right),
$$

then,

$$
\mathcal{P}\left(\tilde{\boldsymbol{k}}_{1}-\tilde{\boldsymbol{k}}_{2}\right)=\mathcal{P}_{t}\left(k_{1}-k_{2}\right) \mathcal{P}_{\perp}\left(\boldsymbol{k}_{1}-\boldsymbol{k}_{2}\right),
$$


with

$$
\begin{gathered}
\mathcal{P}_{t}\left(k_{1}-k_{2}\right)=\int p_{t}(t) e^{i c\left(k_{1}-k_{2}\right) t} d t, \\
\mathcal{P}_{\perp}\left(\boldsymbol{k}_{1}-\boldsymbol{k}_{2}\right)=\int p_{\perp}\left(\boldsymbol{r}_{\perp}\right) e^{i\left(\boldsymbol{k}_{1}-\boldsymbol{k}_{2}\right) \cdot \boldsymbol{r}_{\perp}} d \boldsymbol{r}_{\perp},
\end{gathered}
$$

where

$$
\boldsymbol{r}_{\perp}=(x, y, 0), \quad d \boldsymbol{r}_{\perp}=d x d y .
$$

The width of $\mathcal{P}_{t}\left(k_{1}-k_{2}\right)$ as a function of $k_{1}-k_{2}$ is of the order of $1 /\left(c \sigma_{t}\right)$. If $\mathcal{P}_{t}\left(k_{1}-k_{2}\right)$ is much narrower than any other scale in the problem, such as the width of the radiation spectrum, or the monochromator's FWHM (if used), then we can use the following relation employing the Dirac delta function,

$$
\left|\mathcal{P}_{t}\left(k_{1}-k_{2}\right)\right|^{2}=\kappa_{t} \delta\left(k_{1}-k_{2}\right),
$$

with

$$
\kappa_{t}=(2 \pi / c) \int p_{t}^{2}(t) d t
$$

the derivation is provided in Appendix E.

Using Eqs. (42) to (48), Eq. (41) takes the form

$$
\frac{1}{M}=\left(1-1 / n_{e}\right) \frac{\kappa_{t} \int d k d \Omega_{1} d \Omega_{2} k^{4} \eta_{k n_{1}} I_{k n_{1}}^{(1)} \eta_{k n_{2}} I_{k n_{2}}^{(1)}\left|\mathcal{P}_{\perp}\left(k \boldsymbol{n}_{1}-k \boldsymbol{n}_{2}\right)\right|^{2}}{\left(\int d \boldsymbol{k} \eta_{\boldsymbol{k}} I_{\boldsymbol{k}}^{(1)}\right)^{2}},
$$

where $\boldsymbol{n}_{1}$ and $\boldsymbol{n}_{2}$ are two unit vectors,

$$
\begin{gathered}
\boldsymbol{n}_{i}=\left(n_{i x}, n_{i y}, n_{i z}\right), \quad n_{i z}=\sqrt{1-n_{i x}^{2}-n_{i y}^{2}}, \\
d \Omega_{i}=d n_{i x} d n_{i y} / \sqrt{n_{i x}^{2}+n_{i y}^{2}},
\end{gathered}
$$

where $i=1,2 ; d \Omega_{i}$ is the element of solid angle. Note that the above derivation did not use the paraxial approximation, $\boldsymbol{n}_{i} \approx\left(\phi_{i x}, \phi_{i y}, 1\right) ; \phi_{i x}, \phi_{i y} \ll 1$. However, it can be used in most practical cases. Also, usually $n_{e} \gg 1$ and, hence, $1 / n_{e}$ can be neglected.

For a Gaussian electron bunch,

$$
p(\boldsymbol{r})=\frac{1}{(2 \pi)^{3 / 2} \sigma_{x} \sigma_{y} \sigma_{t}} \exp \left(-\frac{x^{2}}{2 \sigma_{x}^{2}}-\frac{y^{2}}{2 \sigma_{y}^{2}}-\frac{t^{2}}{2 \sigma_{t}^{2}}\right),
$$

and

$$
\begin{gathered}
\left|\mathcal{P}_{\perp}\left(k \boldsymbol{n}_{1}-k \boldsymbol{n}_{2}\right)\right|^{2}=e^{-k^{2} \sigma_{x}^{2}\left(n_{1 x}-n_{2 x}\right)^{2}-k^{2} \sigma_{y}^{2}\left(n_{1 y}-n_{2 y}\right)^{2}}, \\
\kappa_{t}=\frac{\sqrt{\pi}}{c \sigma_{t}} .
\end{gathered}
$$

While in a typical electron storage ring the longitudinal momentum distribution is close to Gaussian, the longitudinal density distribution may not be Gaussian due to beam interaction with its environment (see, for example [24]). This is also the case in IOTA. Equation (48) correctly accounts for the actual longitudinal distribution and it will be used during the proposed experimental study in IOTA. However, to simplify our estimations in Secs. V and VI, we will use Eq. (54).

In the numerical examples in this paper (the Brookhaven experiment [1], and in the proposed experiment in IOTA
$[17,18])$ we use the full version of Eq. (49) and perform numerical integration, because the values of the parameters in these experiments do not allow to use any approximations or simplifications in the integrals. We use the expressions for spectral-angular intensity distribution of wiggler (undulator) radiation from Ref. [25]. Based on these expressions, we developed a PYTHON package for calculation of $I_{k}^{(1)}$, which can be found in the code repository [26]. The developed scripts for numerical integration in Eq. (49) are available in the code repository [27].

Equations (40) and (49) do not reveal the exact distribution of $\mathcal{N}$, they only give the variance $\operatorname{var}(\mathcal{N})$. However, the form of the distribution can be suggested by a simple qualitative argument when the number of longitudinal modes $M_{L}$ (see [7][p. 28]) is much larger than one. For bending-magnet radiation $M_{L} \sim \omega \sigma_{t}$, for undulator radiation $M_{L} \sim \omega \sigma_{t} / N_{u}$, where $N_{\mathrm{u}}$ is the number of undulator periods, see Eq. (56). Indeed, in this case the total number of detected photons $\mathcal{N}$ is the sum of a large number of independent random numbers of detected photons coming from small longitudinal slices of the bunch. Therefore, according to the central limit theorem, $\mathcal{N}$ follows a normal distribution with good accuracy. More details on the exact distribution of $\mathcal{N}$ can be found in [3,7,23,28-30] which suggest that, in the case of incoherent spontaneous radiation, the classical radiated power obeys Gamma statistics.

\section{PRACTICAL APPROXIMATIONS}

The integrals in Eq. (49) can be evaluated analytically in multiple cases, when some approximations are used. Assuming a Gaussian form of $\eta_{k} I_{k}^{(1)}$, an expression for $M$ was previously reported in [2]. Similar calculations for a one-dimensional model (longitudinal) were carried out in [[7] pp. 26-28]. Below we present the results of our 
calculation for the fundamental harmonic of undulator radiation. We assume a small undulator parameter, $K_{\mathrm{u}} \ll 1$, and a large number of periods, $N_{\mathrm{u}} \gg 1$. The electrons are ultrarelativistic, $\gamma \gg 1$. We employ the formula for spectral-angular distribution of the radiation reported in Refs. [31,32]. We consider an ideal detector with a rectangular aperture. The horizontal and vertical angular sizes are $\theta_{x}$ and $\theta_{y}$, respectively. The detector is assumed to be much smaller than the characteristic angular size of the undulator radiation,

$$
\theta_{x}, \theta_{y} \ll 1 /\left(\gamma \sqrt{N_{\mathrm{u}}}\right)
$$

We may assume a detector registering both polarization components. However, given Eq. (55), the vertical component is negligible.

Given the above approximations, the following two expressions for the number of coherent modes $M$ can be obtained in two opposite limiting cases. In the first case, when $\theta_{x}, \theta_{y} \ll 1 /\left(k_{1} \sigma_{x}\right), 1 /\left(k_{1} \sigma_{y}\right)$,

$$
M=\left(1-1 / n_{e}\right)^{-1} \frac{3}{2 \sqrt{\pi}} \frac{c \sigma_{t} k_{1}}{N_{\mathrm{u}}},
$$

which essentially implies radiation, fully coherent in transverse plane. Hence, $M$ is determined solely by the number of longitudinal modes.

In the second case, when $\theta_{x}, \theta_{y} \gg 1 /\left(k_{1} \sigma_{x}\right), 1 /\left(k_{1} \sigma_{y}\right)$,

$$
M=\left(1-1 / n_{e}\right)^{-1} \frac{3}{2 \pi^{3 / 2}} \frac{c \sigma_{t} k_{1}}{N_{\mathrm{u}}}\left(\sigma_{x} k_{1} \theta_{x}\right)\left(\sigma_{y} k_{1} \theta_{y}\right),
$$

where we can see the product of the numbers of both longitudinal and transverse modes. In Eqs. (56) and (57), $k_{1}$ refers to the first harmonic (fundamental) of the undulator radiation. Do not confuse with $\boldsymbol{k}_{1}$, which was used as an integration variable previously. In the limit of $K_{\mathrm{u}} \ll 1$,

$$
k_{1}=2 \gamma^{2} \frac{2 \pi}{\lambda_{\mathrm{u}}}
$$

where $\lambda_{\mathrm{u}}$ is the undulator period.

Another example when an expression for $M$ takes a simple form is the extreme approximation of a single mode detector, which can only sense one optical mode with a certain wave vector $\boldsymbol{k}$. It readily follows from Eq. (41) that in this model $M=1 /\left(1-1 / n_{e}\right)$. Hence,

$$
\operatorname{var}(\mathcal{N})=\langle\mathcal{N}\rangle+\left(1-1 / n_{e}\right)\langle\mathcal{N}\rangle^{2}
$$

which coincides with the results, reported in Refs. [1,3336]. Equation (59) is correct for any source of temporally incoherent synchrotron radiation, and for any direction and wavelength of $\boldsymbol{k}$.

\section{BROOKHAVEN EXPERIMENT}

In the seminal experiment at Brookhaven National Laboratory [1], the fluctuations in the wiggler and bending-magnet radiation were studied in the vacuumultraviolet (VUV) electron storage ring. The wiggler radiation data in Fig. 3 were extracted from the original paper [1] by digitizing the plot. The scale was also changed from log-log to a linear scale. This procedure could have introduced some deviations from the original data, but the deviations are believed to be negligible.

The data for the wiggler radiation was collected for the fundamental harmonic, $\lambda_{1}=532 \mathrm{~nm}$. An optical interference filter with $\mathrm{FWHM}=3.2 \mathrm{~nm}$ and a maximum transmission at $\lambda_{1}$ was used. Polarizing filters were not used. The parameters of the wiggler and the electron bunch are listed in Table I. A silicon PIN photodiode was used to detect the wiggler radiation. The measurements were carried out with two different lattice configurations, i.e., two different transverse beam profiles-in the language of the authors of [1], a tightly focused beam and a loosely focused beam. In this paper, we will refer to them as lattice configuration $\mathrm{A}$ and lattice configuration $\mathrm{B}$, respectively.

The mean photoelectron count was mainly varied by using a variable neutral density filter. For filters of this type, the efficiency $\eta_{k}$ does not depend on the radiation wavelength. Hence, $\eta_{k_{1}}, \eta_{k_{2}}$, and $\eta_{k}$ cancel out in the numerator and the denominator of Eq. (49). In Eq. (40), $M$ does not change when the transmission of the neutral density filter changes. However, the average number of detected photons $\langle\mathcal{N}\rangle$ changes, in accordance with Eq. (38).

The two solid curves in Fig. 3 are the theoretical predictions made by Eqs. (40) and (49) using the parameters of the electron bunch, the wiggler, and the monochromator, given in [1]. The Gaussian model for the transmission of the monochromator was used. Equation (49) gives the following results for lattice configurations $\mathrm{A}$ and $\mathrm{B}$, $M_{A}=5.7 \times 10^{4}, M_{B}=6.0 \times 10^{4}$.

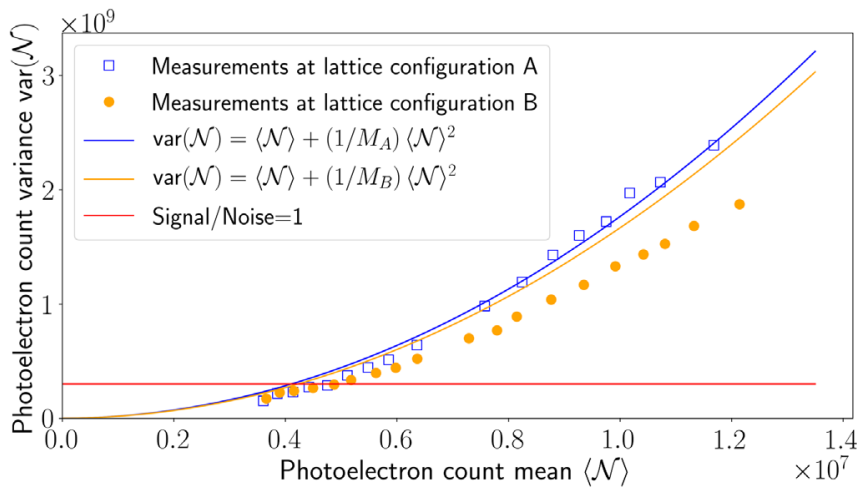

FIG. 3. Experimental data from Ref. [1] for wiggler radiation (points) and predictions made by our calculation (solid curves). The noise variance $\left(3 \times 10^{8}\right)$ has been subtracted from the data. 
TABLE I. Summary of the parameters of the Brookhaven experiment [1] and the proposed experiment at Fermilab in the IOTA storage ring $[16,18]$. The electron bunch dimensions correspond to the center of the wiggler (undulator). Both rings store a single electron bunch. The parameters of IOTA are given at $I_{\text {beam }}=1.3 \mathrm{~mA}$. For more details about the undulator in IOTA, see [37]. Some parameters of the BNL VUV ring are followed by (A) or (B) to specify the corresponding lattice configuration.

\begin{tabular}{lcc}
\hline \hline Parameter & BNL VUV ring & Fermilab IOTA ring \\
\hline Ring circumference & $51.06 \mathrm{~m}(170.2 \mathrm{~ns})$ & $40 \mathrm{~m}(133 \mathrm{~ns})$ \\
Beam energy & $650 \mathrm{MeV}$ & $100 \mathrm{MeV}$ \\
Typical average current & $50 \mathrm{~mA}$ & $1.3 \mathrm{~mA}$ \\
Horizontal emittance $(\mathrm{rms}), \epsilon_{x}$ & $0.80 \mu \mathrm{m}(\mathrm{A}), 0.74 \mu \mathrm{m}(\mathrm{B})$ & $0.32 \mu \mathrm{m}$ \\
Vertical emittance $(\mathrm{rms}), \epsilon_{y}$ & $0.20 \mu \mathrm{m}(\mathrm{A}), 0.26 \mu \mathrm{m}(\mathrm{B})$ & $31 \mathrm{~nm}$ \\
Relative momentum spread, $\sigma_{p} / p$ & $(\mathrm{not}$ available $)$ & $3.1 \times 10^{-4}$ \\
Horizontal beam size, $\sigma_{x}$ & $1.0 \mathrm{~mm}(\mathrm{~A}), 0.96 \mathrm{~mm}(\mathrm{~B})$ & $815 \mu \mathrm{m}$ \\
Vertical beam size, $\sigma_{y}$ & $0.32 \mathrm{~mm}(\mathrm{~A}), 0.36 \mathrm{~mm}(\mathrm{~B})$ & $75 \mu \mathrm{m}$ \\
Longitudinal bunch size, $\sigma_{z}\left(\sigma_{t}\right)$ & $6.11 \mathrm{~cm}(0.204 \mathrm{~ns})$ & $38 \mathrm{~cm}(1.3 \mathrm{~ns})$ \\
Beam lifetime & $\approx 100 \mathrm{~min}$ & $>10 \mathrm{~min}$ \\
Wiggler (undulator) parameter, $K_{\mathrm{u}}$ & 5.7 & 1.06 \\
Wiggler (undulator) period, $\lambda_{\mathrm{u}}$ & $10 \mathrm{~cm}$ & $5.5 \mathrm{~cm}$ \\
Number of wiggler (undulator) periods, $N_{\mathrm{u}}$ & 22.5 & 10.5 \\
Fundamental harmonic wavelength, $\lambda_{1}$ & $532 \mathrm{~nm}$ & $1120 \mathrm{~nm}$ \\
Photodiode diameter & $(\mathrm{not}$ available $)$ & $1 \mathrm{~mm}$ \\
Quantum efficiency @ $\lambda_{1}$ & $78 \%$ & $80 \%$ \\
Monochromator FWHM & $3.2 \mathrm{~nm}$ & $($ not used) \\
Simulated number of photon counts per turn, $\langle\mathcal{N}\rangle$ & $5.6 \times 10^{7}$ & $3.3 \times 10^{7}$ \\
Simulated number of coherent modes, $M$ & $\approx 1.2 \times 10^{7}$ & $\approx 3$ \\
Ratio of fluctuation contributions, $\langle\mathcal{N}\rangle / M$ & $5.7 \times 10^{4}(\mathrm{~A}), 6.0 \times 10^{4}(\mathrm{~B})$ & \\
\hline \hline
\end{tabular}

Although our calculation agrees well with the measurements at lattice configuration $\mathrm{A}$, there is some disagreement at lattice configuration $\mathrm{B}$. In terms of the $M$ parameter, the deviation is about $20 \%$. It is not clear if this disagreement comes from inaccuracy in our calculations or from our incomplete understanding of the experiment configuration from [1]. The experiment took place three decades ago and a detailed description is currently not available. This is one of the motivations for carrying out a new dedicated experiment in IOTA.

Furthermore, in the Brookhaven experiment, the number of coherent modes $\left(M_{A}, M_{B}\right)$ is much smaller than the average number of photoelectrons $\langle\mathcal{N}\rangle$. Hence, the first term in Eq. (40) is negligible. Therefore, these data do not test Eq. (40) in the regime when the two contributions are comparable. This may be achievable in an independent experiment in IOTA.

\section{PROPOSED EXPERIMENT IN IOTA}

We propose a dedicated study of fluctuations in the number of detected undulator radiation photons in the IOTA storage ring at Fermilab [16].

In addition to the motivation outlined in the end of Sec. V, these measurements can also provide information about the dimensions and the shape of the electron bunch in IOTA. Indeed, Eqs. (49), (53), and (54) indicate that the magnitude of the fluctuations depends on the dimensions of a Gaussian bunch. Also, if the longitudinal shape of the bunch is not Gaussian, it will be reflected in the magnitude of the fluctuations according to Eq. (48).

Currently, the transverse beam sizes $\sigma_{x}$ and $\sigma_{y}$ are measured by synchrotron radiation monitors using the radiation from dipole magnets, while $\sigma_{t}$ is measured by a wall-current monitor. The existing physical model of beam lifetimes and beam shapes in IOTA (taking into account intrabeam scattering, gas scattering, quantum excitation, etc.) is not fully consistent with observations [38]. An independent estimate of $\sigma_{x}, \sigma_{y}$, and $\sigma_{t}$ through fluctuations in undulator radiation, see Eq. (49), may help resolve inconsistencies.

Because in IOTA the fundamental of the undulator radiation is in the near infrared range, $\lambda_{1}=1120 \mathrm{~nm}$, it will be necessary to use an InGaAs PIN photodiode (for example, [39]) instead of the silicon one, used in [1]. We will not use a polarizing filter. Both polarization components will be registered. The spectral distribution of the number of photons emitted into an aperture with 2 inch diameter, located $3.5 \mathrm{~m}$ away from the undulator in IOTA is shown in Fig. 4 (the red curve). This curve was obtained by using our simulation package [26] and also verified by an independent simulation using the SRW software [40]. This curve represents the intensity per electron.

Assuming no additional losses apart from the quantum efficiency of the InGaAs photodiode, we estimated the expected number of photon counts $\langle\mathcal{N}\rangle$ and the number of coherent modes $M$ for the proposed IOTA experiment. The results are given in Table I and in Fig. 5. We propose not to 


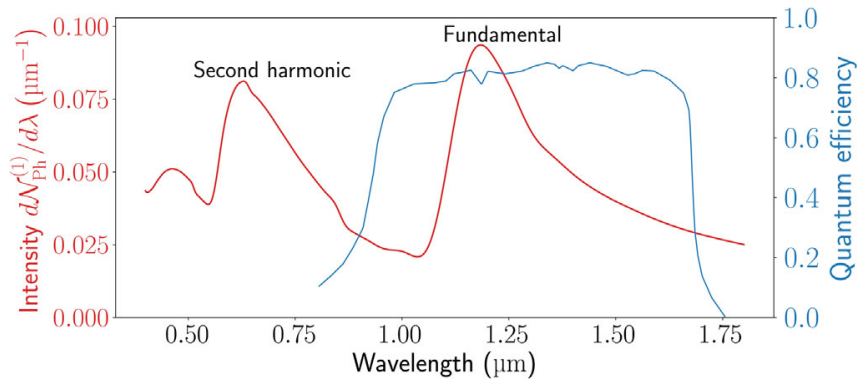

FIG. 4. Spectral distribution of the number of emitted photons (per electron) from the undulator in IOTA (red curve). Quantum efficiency of an InGaAs PIN photodiode (blue curve).

use a monochromator in IOTA. This will let us see approximately the same average photon count $\langle\mathcal{N}\rangle$ as in the Brookhaven experiment, even though the beam current, beam energy, wiggler (undulator) parameter $K_{\mathrm{u}}$, and the number of wiggler (undulator) periods is lower in IOTA. This is important in order to have a signal of a comfortable amplitude for measurements.

The absence of a monochromator may also make the number of coherent modes $M$ much larger than in the Brookhaven experiment, see Table I, and comparable to $\langle\mathcal{N}\rangle$. This may allow us to probe Eq. (40) in the regime where the two sum terms are comparable, see the last row in Table I. Indeed, the curve denoted by $M_{0}$ in Fig. 5 is rather close to the green dashed line, representing the photon shot noise fluctuations. $M_{0}$ was calculated by Eq. (49) assuming no additional losses except for the quantum efficiency of the detector. This is the value of $M$ reported in Table I. The curves denoted by $M_{1}$ and $M_{2}$ were calculated with bandpass filters $(1050 \mathrm{~nm}, 1350 \mathrm{~nm})$ and $(1150 \mathrm{~nm}$, $1300 \mathrm{~nm}$ ), respectively: $M_{1}=4.9 \times 10^{6}$ and $M_{2}=2.7 \times 10^{6}$. We provide these two curves to show how the data may change due to spectral properties of additional optical elements in the measurement apparatus. The measured spectral range will likely be narrower than that of the InGaAs photodiode, and it will be determined by

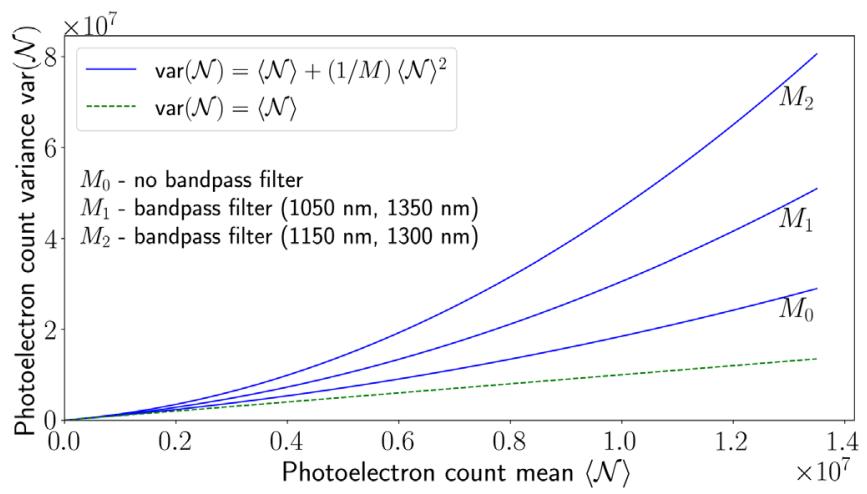

FIG. 5. Simulations for photoelectron count variance in IOTA for three different filter configurations. The green dashed line represents the photon shot noise contribution to the fluctuations. transmission curves of additional optical elements, such as lenses, mirrors, and, possibly, bandpass filters.

As opposed to neutral density filters, bandpass filters do change the number of coherent modes $M$. However, typically, at fixed beam current and shape, the ratio between the incoherence contribution and the photon shot noise contribution in Eq. (40), namely, $\langle\mathcal{N}\rangle / M$, stays approximately constant when different bandpass filters are used. In a simplified model of Gaussian spectral-angular distribution of radiation, considered in [2], this statement is exact. In the proposed experiment in IOTA, at $I_{\text {beam }}=$ $1.3 \mathrm{~mA},\langle\mathcal{N}\rangle / M \approx 3$, see Table I.

In the simulation in Fig. 5, we assume the same measurement procedure as in the Brookhaven experiment. Namely, in each curve, the mean photon count $\langle\mathcal{N}\rangle$ is varied by using a variable neutral density filter. The bunch charge is constant. This means that $\sigma_{x}, \sigma_{y}, \sigma_{t}$, and, hence, $M$ are constant too. The values used in the simulation are provided in Table I. In our experiment in IOTA, we also plan to vary the bunch charge and observe how the magnitude of the fluctuations $\operatorname{var}(\mathcal{N})$ changes. The dependence of $\operatorname{var}(\mathcal{N})$ on $\langle\mathcal{N}\rangle$ is expected to be nontrivial (not a parabola) because the dimensions of the electron bunch in IOTA change significantly with beam current, and so should $M$, according to Eq. (49). Some examples of the effects that depend on beam current and that determine the electron bunch shape and size in IOTA include intrabeam scattering [41-43] and longitudinal self-focusing due to space-charge [24]. The proposed measurements may help improve our understanding of these effects in IOTA.

\section{CONCLUSIONS}

We derived Eq. (34), which predicts the fluctuations $\operatorname{var}(\mathcal{N})$ in spontaneous synchrotron radiation with any degree of coherence for an electron bunch of arbitrary shape. Then, several possible simplifications were considered in the case of temporally incoherent radiation. The formulas properly take into account the discrete nature of light and the quantum efficiency of the detector, which, in general, is a function of the radiation wavelength. A spectral filter with any transmission function can be incorporated by including the transmission function into $\eta_{k}$ in Eqs. (34) and (49). The detector acceptance can be taken into account by setting $\eta_{k}$ to zero outside of a given angular range.

The predicted variance vs radiation intensity was compared with the empirical data from a previous experiment at Brookhaven [1] for the case of wiggler radiation with a dominant incoherence contribution. We propose a new experimental study of undulator radiation fluctuations in IOTA. In this experiment, Eq. (40) may be tested in a new regime, when the photon shot noise contribution and the incoherence contribution are comparable.

As was pointed out in [2-5], the fluctuations in synchrotron radiation can be used to determine the bunch 
length on a picosecond scale, and the proof of principle experiments were successful. In IOTA, the longitudinal bunch size is relatively large, $\sigma_{z}=38 \mathrm{~cm}$ at $I_{\text {beam }}=1.3 \mathrm{~mA}$, and can be easily measured with a wall-current monitor. On the other hand, the transverse bunch size (especially vertical) can be quite small, down to a few tens of microns, where it may be difficult to measure by conventional synchrotron radiation monitors, because of the diffraction limit. However, the number of coherent modes $M$ in the undulator radiation in IOTA is rather sensitive to the transverse bunch size. Therefore, the magnitude of the fluctuations may help determine the transverse dimensions of the electron bunch.

In any case, the measurement of undulator radiation fluctuations in IOTA is a measurement of a certain function [see Eqs. (40) and (49)] of electron bunch dimensions and shape. This measurement is independent from the wallcurrent monitor and the synchrotron radiation monitors. Therefore, it may help refine the existing model of the physical effects determining the electron bunch parameters in IOTA. The results of the proposed experiment in IOTA will be reported in a separate paper.

\section{ACKNOWLEDGMENTS}

One of the coauthors (A. H.) is grateful to C. Pellegrini, G. Stupakov, and Y. Cai (SLAC) for many in-depth physics discussions on the subject. This research is supported by the University of Chicago and the U.S. Department of Energy under Contracts No. DE-AC02-76SF00515 and No. DE-AC02-06CH11357. This manuscript has been authored by Fermi Research Alliance, LLC under Contract No. DE-AC02-07CH11359 with the U.S. Department of Energy, Office of Science, Office of High Energy Physics.

\section{APPENDIX A: BEAM SPLITTER DETECTOR MODEL}

Using Eq. (12) in Eq. (14) we obtain

$$
\hat{\mathcal{N}}=\sum_{k}\left(\eta_{k} \hat{a}_{k}^{\dagger} \hat{a}_{k}+i \sqrt{\eta_{k}} \sqrt{1-\eta_{k}} \hat{a}_{k}^{\dagger} \hat{c}_{k}-i \sqrt{\eta_{k}} \sqrt{1-\eta_{k}} \hat{c}_{k}^{\dagger} \hat{a}_{k}+\left(1-\eta_{k}\right) \hat{c}_{k}^{\dagger} \hat{c}_{k}\right)
$$

Equation (18) readily follows from Eq. (A1), since only the first term in the parentheses will provide a nonzero contribution.

The derivation of Eq. (19) is more lengthy,

$$
\begin{aligned}
{ }_{c}\left\langle 0\left|\hat{\mathcal{N}}^{2}\right| 0\right\rangle_{c}= & { }_{c}\left\langle 0\left|\sum_{\boldsymbol{k}_{1}} \hat{b}_{\boldsymbol{k}_{1}}^{\dagger} \hat{b}_{\boldsymbol{k}_{1}} \sum_{\boldsymbol{k}_{2}} \hat{b}_{\boldsymbol{k}_{2}}^{\dagger} \hat{b}_{\boldsymbol{k}_{2}}\right| 0\right\rangle_{c} \\
= & { }_{c}\langle 0| \sum_{\boldsymbol{k}_{1}, \boldsymbol{k}_{2}}\left(\eta_{\boldsymbol{k}_{1}} \hat{a}_{\boldsymbol{k}_{1}}^{\dagger} \hat{a}_{\boldsymbol{k}_{1}}+i \sqrt{\eta_{\boldsymbol{k}_{1}}} \sqrt{1-\eta_{\boldsymbol{k}_{1}}} \hat{a}_{\boldsymbol{k}_{1}}^{\dagger} \hat{c}_{\boldsymbol{k}_{1}}-i \sqrt{\eta_{\boldsymbol{k}_{1}}} \sqrt{1-\eta_{\boldsymbol{k}_{1}}} \hat{c}_{\boldsymbol{k}_{1}}^{\dagger} \hat{a}_{\boldsymbol{k}_{1}}+\left(1-\eta_{\boldsymbol{k}_{1}}\right) \hat{c}_{\boldsymbol{k}_{1}}^{\dagger} \hat{c}_{\boldsymbol{k}_{1}}\right) \\
& \left(\eta_{\boldsymbol{k}_{2}} \hat{a}_{\boldsymbol{k}_{2}}^{\dagger} \hat{a}_{\boldsymbol{k}_{2}}+i \sqrt{\eta_{\boldsymbol{k}_{2}}} \sqrt{1-\eta_{\boldsymbol{k}_{2}}} \hat{a}_{\boldsymbol{k}_{2}}^{\dagger} \hat{c}_{\boldsymbol{k}_{2}}-i \sqrt{\eta_{\boldsymbol{k}_{2}}} \sqrt{1-\eta_{\boldsymbol{k}_{2}}} \hat{c}_{\boldsymbol{k}_{2}}^{\dagger} \hat{a}_{\boldsymbol{k}_{2}}+\left(1-\eta_{\boldsymbol{k}_{2}}\right) \hat{c}_{\boldsymbol{k}_{2}}^{\dagger} \hat{c}_{\boldsymbol{k}_{2}}\right)|0\rangle_{c} .
\end{aligned}
$$

After considering the products of each term from the first parentheses with each term from the second parentheses, we conclude that only the following two will provide nonzero contributions,

$$
\begin{aligned}
{ }_{c}\left\langle 0\left|\hat{\mathcal{N}}^{2}\right| 0\right\rangle_{c} & ={ }_{c}\left\langle 0\left|\sum_{\boldsymbol{k}_{1}, \boldsymbol{k}_{2}}\left(\eta_{\boldsymbol{k}_{1}} \eta_{\boldsymbol{k}_{2}} \hat{a}_{\boldsymbol{k}_{1}}^{\dagger} \hat{a}_{\boldsymbol{k}_{1}} \hat{a}_{\boldsymbol{k}_{2}}^{\dagger} \hat{a}_{\boldsymbol{k}_{2}}+\sqrt{\eta_{\boldsymbol{k}_{1}}\left(1-\eta_{\boldsymbol{k}_{1}}\right) \eta_{\boldsymbol{k}_{2}}\left(1-\eta_{\boldsymbol{k}_{2}}\right)} \hat{a}_{\boldsymbol{k}_{1}}^{\dagger} \hat{c}_{\boldsymbol{k}_{1}} \hat{c}_{\boldsymbol{k}_{2}}^{\dagger} \hat{a}_{\boldsymbol{k}_{2}}\right)\right| 0\right\rangle_{c} \\
& =\sum_{\boldsymbol{k}_{1}, \boldsymbol{k}_{2}} \eta_{\boldsymbol{k}_{1}} \eta_{\boldsymbol{k}_{2}} \hat{a}_{\boldsymbol{k}_{1}}^{\dagger}\left(\hat{a}_{\boldsymbol{k}_{2}}^{\dagger} \hat{a}_{\boldsymbol{k}_{1}}+\delta_{\boldsymbol{k}_{1}, \boldsymbol{k}_{2}}\right) \hat{a}_{\boldsymbol{k}_{2}}+\sum_{\boldsymbol{k}} \eta_{\boldsymbol{k}}\left(1-\eta_{\boldsymbol{k}}\right) \hat{a}_{\boldsymbol{k}}^{\dagger} \hat{a}_{\boldsymbol{k}} \\
& =\sum_{\boldsymbol{k}_{1}, \boldsymbol{k}_{2}} \eta_{\boldsymbol{k}_{1}} \eta_{\boldsymbol{k}_{2}} \hat{a}_{\boldsymbol{k}_{1}}^{\dagger} \hat{a}_{\boldsymbol{k}_{2}}^{\dagger} \hat{a}_{\boldsymbol{k}_{1}} \hat{a}_{\boldsymbol{k}_{2}}+\sum_{\boldsymbol{k}} \eta_{\boldsymbol{k}}^{2} \hat{a}_{\boldsymbol{k}}^{\dagger} \hat{a}_{\boldsymbol{k}}+\sum_{\boldsymbol{k}} \eta_{\boldsymbol{k}} \hat{a}_{\boldsymbol{k}}^{\dagger} \hat{a}_{\boldsymbol{k}}-\sum_{\boldsymbol{k}} \eta_{\boldsymbol{k}}^{2} \hat{a}_{\boldsymbol{k}}^{\dagger} \hat{a}_{\boldsymbol{k}},
\end{aligned}
$$

where we employed the commutation relation [14],

$$
\left[\hat{a}_{\boldsymbol{k}_{1}}, \hat{a}_{\boldsymbol{k}_{2}}^{\dagger}\right]=\delta_{\boldsymbol{k}_{1}, \boldsymbol{k}_{2}}= \begin{cases}1, & \text { if } \boldsymbol{k}_{1}=\boldsymbol{k}_{2} \\ 0, & \text { otherwise }\end{cases}
$$

and also the fact that

$$
{ }_{c}\left\langle 0\left|\hat{c}_{\boldsymbol{k}_{1}} \hat{c}_{\boldsymbol{k}_{2}}^{\dagger}\right| 0\right\rangle_{c}=\delta_{\boldsymbol{k}_{1}, \boldsymbol{k}_{2}} .
$$

Clearly, Eq. (A3) is equivalent to Eq. (19). 


\section{APPENDIX B: CALCULATION OF THE EIGENVALUES OF THE COHERENT STATES OF RADIATION OF AN ELECTRON BUNCH}

The current density of the electron bunch is

$$
\boldsymbol{j}(\xi, \boldsymbol{r}, t)=\sum_{m} e \boldsymbol{v}_{m}(t) \delta\left(\boldsymbol{r}_{m}(t)-\boldsymbol{r}\right),
$$

where $\delta(.$.$) is the Dirac delta function, m=1, \ldots n_{e}$.

Without loss of generality one can choose the electron with $m=1$ to be the reference electron. To be specific, for this electron, the time of entrance is zero, $t_{1}=0$, and the initial transverse position is zero, $\boldsymbol{r}_{1 \perp}=0$. We assume that in the synchrotron light source under consideration the trajectory of the $m$ th electron is merely the trajectory of the reference electron offset by $\boldsymbol{r}_{m \perp}=\left(x_{m}, y_{m}, 0\right)$. It is usually a good approximation in undulators, wigglers, and bending magnets. In this case,

$\boldsymbol{j}(\xi, \boldsymbol{r}, t)=\sum_{m} e \boldsymbol{v}_{1}\left(t-t_{m}\right) \delta\left(\boldsymbol{r}_{1}\left(t-t_{m}\right)+\boldsymbol{r}_{m \perp}-\boldsymbol{r}\right)$.

By integrating over $\boldsymbol{r}$ in Eq. (10) using Eq. (B2), we obtain

$$
\begin{aligned}
\alpha_{k}(\xi)= & \frac{i e}{\sqrt{2 L^{3} \hbar \omega}} \sum_{m} \int d t \boldsymbol{e}_{s}(\boldsymbol{k}) \cdot \boldsymbol{v}_{1}\left(t-t_{m}\right) \\
& \times e^{i \boldsymbol{k} \cdot \boldsymbol{r}_{1}\left(t-t_{m}\right)+i \boldsymbol{k} \cdot r_{m \perp}-i \omega\left(t-t_{m}\right)} .
\end{aligned}
$$

By changing the integration variable from $t$ to $t-t_{m}$ in each summation term one arrives at

$$
\begin{aligned}
\alpha_{\boldsymbol{k}}(\xi) & =\frac{i e}{\sqrt{2 L^{3} \hbar \omega}} \sum_{m} e^{i \boldsymbol{k} \cdot \boldsymbol{r}_{m \perp}-i \omega t_{m}} \int d t \boldsymbol{e}_{s}(\boldsymbol{k}) \cdot \boldsymbol{v}_{1}(t) e^{i \boldsymbol{k} \cdot \boldsymbol{r}_{1}(t)-i \omega t} \\
& =\left(\sum_{m} e^{i \tilde{k} \cdot r_{m}}\right) \times \alpha_{k}^{(1)} .
\end{aligned}
$$

Similar calculations have been presented in $[2,7,19]$.

\section{APPENDIX C: CALCULATION OF PHOTOCOUNT VARIANCE FOR ARBITRARY BUNCH SHAPE}

In this Appendix, we derive Eq. (34). We will need to use the following relation in Eq. (27),

$$
\begin{aligned}
\mathcal{J}_{\boldsymbol{k}_{1}}(\xi) \mathcal{J}_{\boldsymbol{k}_{2}}(\xi) & =\left|\sum_{m} e^{i \tilde{\boldsymbol{k}}_{1} \cdot \boldsymbol{r}_{m}}\right|^{2}\left|\sum_{n} e^{i \tilde{\boldsymbol{k}}_{2} \cdot \boldsymbol{r}_{n}}\right|^{2}=\left(n_{e}+\sum_{m \neq n} e^{i \tilde{\boldsymbol{k}}_{1} \cdot\left(\boldsymbol{r}_{m}-\boldsymbol{r}_{n}\right)}\right)\left(n_{e}+\sum_{i \neq j} e^{-i \tilde{\boldsymbol{k}}_{2} \cdot\left(\boldsymbol{r}_{i}-\boldsymbol{r}_{j}\right)}\right) \\
& =n_{e}^{2}+n_{e} \sum_{m \neq n} e^{i \tilde{\boldsymbol{k}}_{1} \cdot\left(\boldsymbol{r}_{m}-\boldsymbol{r}_{n}\right)}+n_{e} \sum_{i \neq j} e^{-i \tilde{\boldsymbol{k}}_{2} \cdot\left(\boldsymbol{r}_{i}-\boldsymbol{r}_{j}\right)}+\sum_{m \neq n} e^{i\left(\tilde{\boldsymbol{k}}_{1}-\tilde{\boldsymbol{k}}_{2}\right) \cdot\left(\boldsymbol{r}_{m}-\boldsymbol{r}_{n}\right)}+\sum_{\substack{m \neq n, i \neq j \\
m, n \neq i, j}} e^{i \tilde{\boldsymbol{k}}_{1}\left(\boldsymbol{r}_{m}-\boldsymbol{r}_{n}\right)-i \tilde{\boldsymbol{k}}_{2}\left(\boldsymbol{r}_{i}-\boldsymbol{r}_{j}\right)} .
\end{aligned}
$$

The next step is to multiply each term of Eq. (C1) by $p(\xi)$ and integrate over $\xi$. The integral of the first term in Eq. (C1) is trivial. The second, third and fourth terms have the same form as Eq. (31). Therefore, we simply provide the results,

$$
\begin{aligned}
& \int d \xi p(\xi) n_{e} \sum_{m \neq n} e^{i \tilde{\boldsymbol{k}}_{1} \cdot\left(\boldsymbol{r}_{m}-\boldsymbol{r}_{n}\right)}=n_{e}^{2}\left(n_{e}-1\right)\left|\mathcal{P}\left(\tilde{\boldsymbol{k}}_{1}\right)\right|^{2}, \\
& \int d \xi p(\xi) n_{e} \sum_{i \neq j} e^{-i \tilde{\boldsymbol{k}}_{2} \cdot\left(\boldsymbol{r}_{i}-\boldsymbol{r}_{j}\right)}=n_{e}^{2}\left(n_{e}-1\right)\left|\mathcal{P}\left(\tilde{\boldsymbol{k}}_{2}\right)\right|^{2}, \\
& \int d \xi p(\xi) \sum_{m \neq n} e^{i\left(\tilde{\boldsymbol{k}}_{1}-\tilde{\boldsymbol{k}}_{2}\right) \cdot\left(\boldsymbol{r}_{m}-\boldsymbol{r}_{n}\right)}=n_{e}\left(n_{e}-1\right)\left|\mathcal{P}\left(\tilde{\boldsymbol{k}}_{1}-\tilde{\boldsymbol{k}}_{2}\right)\right|^{2} .
\end{aligned}
$$

The fifth (last) term has to be considered separately. It can be represented in the following form,

$$
\sum_{\substack{m \neq n, i \neq j \\ m, n \neq i, j}} e^{i \tilde{\boldsymbol{k}}_{1}\left(\boldsymbol{r}_{m}-\boldsymbol{r}_{n}\right)-i \tilde{\boldsymbol{k}}_{2}\left(\boldsymbol{r}_{i}-\boldsymbol{r}_{j}\right)}=A_{1}(\xi)+A_{2}(\xi)+A_{3}(\xi),
$$

where

$$
\begin{aligned}
& A_{1}(\xi)=\sum_{\substack{m \neq n \\
j \neq n \\
j \neq m}} e^{i\left(\tilde{\boldsymbol{k}}_{1}-\tilde{\boldsymbol{k}}_{2}\right) \cdot \boldsymbol{r}_{m}-i \tilde{\boldsymbol{k}}_{1} \cdot \boldsymbol{r}_{n}+i \tilde{\boldsymbol{k}}_{2} \cdot \boldsymbol{r}_{j}} \\
& A_{2}(\xi)=\sum_{\substack{m \neq n \\
i \neq n \\
i \neq m}} e^{-i\left(\tilde{\boldsymbol{k}}_{1}-\tilde{\boldsymbol{k}}_{2}\right) \cdot \boldsymbol{r}_{n}+i \tilde{\boldsymbol{k}}_{1} \cdot \boldsymbol{r}_{m}-i \tilde{\boldsymbol{k}}_{2} \cdot \boldsymbol{r}_{i}}, \\
& A_{3}(\xi)=\sum_{\substack{m \neq n \\
i \neq j \\
i \neq n \\
j \neq n}} e^{i \tilde{\boldsymbol{k}}_{1} \cdot\left(\boldsymbol{r}_{m}-\boldsymbol{r}_{n}\right)-i \tilde{\boldsymbol{k}}_{2} \cdot\left(\boldsymbol{r}_{i}-\boldsymbol{r}_{j}\right)} .
\end{aligned}
$$

Integration of Eq. (C5) is analogous to Eq. (31), with the exception that counting the terms in the sums is more difficult. Omitting the details,

$$
\begin{aligned}
\int d \xi p(\xi) A_{1}(\xi)= & {\left[\int d \xi p(\xi) A_{2}(\xi)\right]^{*} } \\
= & n_{e}\left(n_{e}-1\right)\left(n_{e}-2\right) \\
& \times \mathcal{P}\left(\tilde{\boldsymbol{k}}_{1}-\tilde{\boldsymbol{k}}_{2}\right) \mathcal{P}^{*}\left(\tilde{\boldsymbol{k}}_{1}\right) \mathcal{P}\left(\tilde{\boldsymbol{k}}_{2}\right)
\end{aligned}
$$




$$
\int d \xi p(\xi) A_{3}(\xi)=n_{e}\left(n_{e}-1\right)\left(n_{e}^{2}-3 n_{e}+3\right)\left|\mathcal{P}\left(\tilde{\boldsymbol{k}}_{1}\right) \mathcal{P}\left(\tilde{\boldsymbol{k}}_{2}\right)\right|^{2}
$$

When Eqs. (C2) to (C4), (C9) and (C10) are used together with Eq. (C1) to calculate the integral over $\xi$ in Eq. (27), one arrives at

$$
\begin{aligned}
\int d \xi p(\xi) \mathcal{J}_{\boldsymbol{k}_{1}}(\xi) \mathcal{J}_{\boldsymbol{k}_{2}}(\xi)= & n_{e}^{2}+n_{e}\left(n_{e}-1\right)\left|\mathcal{P}\left(\tilde{\boldsymbol{k}}_{1}-\tilde{\boldsymbol{k}}_{2}\right)\right|^{2} \\
& +n_{e}\left(n_{e}-1\right)\left[n_{e}\left(\left|\mathcal{P}\left(\tilde{\boldsymbol{k}}_{1}\right)\right|^{2}+\left|\mathcal{P}\left(\tilde{\boldsymbol{k}}_{2}\right)\right|^{2}\right)+\left(n_{e}-2\right)\left(\mathcal{P}\left(\tilde{\boldsymbol{k}}_{1}-\tilde{\boldsymbol{k}}_{2}\right) \mathcal{P}^{*}\left(\tilde{\boldsymbol{k}}_{1}\right) \mathcal{P}\left(\tilde{\boldsymbol{k}}_{2}\right)+\text { c.c. }\right)\right. \\
& \left.+\left(n_{e}^{2}-3 n_{e}+3\right)\left|\mathcal{P}\left(\tilde{\boldsymbol{k}}_{1}\right) \mathcal{P}\left(\tilde{\boldsymbol{k}}_{2}\right)\right|^{2}\right]
\end{aligned}
$$

Finally, one can obtain Eq. (34), by using Eqs. (33) and (C11) in Eq. (27).

\section{APPENDIX D: GAUSSIAN BUNCH EXAMPLE}

In this Appendix we provide the form of Eqs. (33) and (34) for a Gaussian electron bunch, where each electron has the probability function as in Eq. (52). In this case, the integration over $\boldsymbol{r}$ in Eq. (32) can be performed by using the Hubbard-Stratonovich identity [44]. The results are

$$
\langle\mathcal{N}\rangle=n_{e} \int d \boldsymbol{k} \eta_{\boldsymbol{k}} \boldsymbol{I}_{\boldsymbol{k}}^{(1)}\left(1+\left(n_{e}-1\right) e^{-\boldsymbol{K} \cdot \mathbf{\Sigma}}\right),
$$

where $\mathbf{K} \equiv\left(k_{x}^{2}, k_{y}^{2}, k^{2}\right), \mathbf{\Sigma} \equiv\left(\sigma_{x}^{2}, \sigma_{y}^{2}, c^{2} \sigma_{t}^{2}\right)$,

$$
\operatorname{var}(\mathcal{N})=\langle\mathcal{N}\rangle+n_{e}\left(n_{e}-1\right) \int d \boldsymbol{k}_{1} d \boldsymbol{k}_{2} \eta_{\boldsymbol{k}_{1}} I_{\boldsymbol{k}_{1}}^{(1)} \eta_{\boldsymbol{k}_{2}} I_{\boldsymbol{k}_{2}}^{(1)}\left[\Delta^{2}-\Sigma^{2}+2\left(n_{e}-2\right) \Sigma(\Sigma-\Delta)\right]
$$

where

$$
\begin{gathered}
\Delta=e^{-\frac{1}{2} \mathbf{\Delta}_{12} \cdot \mathbf{\Sigma}}, \quad \Sigma=e^{-\frac{1}{2} \mathbf{K}_{12} \cdot \mathbf{\Sigma}}, \\
\boldsymbol{\Delta}_{12}=\left[\left(k_{1 x}-k_{2 x}\right)^{2},\left(k_{1 y}-k_{2 y}\right)^{2},\left(k_{1}-k_{2}\right)^{2}\right], \\
\mathbf{K}_{12}=\left(k_{1 x}^{2}+k_{2 x}^{2}, k_{1 y}^{2}+k_{2 y}^{2}, k_{1}^{2}+k_{2}^{2}\right) .
\end{gathered}
$$

\section{APPENDIX E: BROAD-SPECTRUM APPROXIMATION}

Start with the following ansatz,

$$
\left|\mathcal{P}_{t}(k)\right|^{2}=\kappa_{t} \delta(k) .
$$

Then,

$$
\begin{aligned}
\kappa_{t} & =\int d k\left|\mathcal{P}_{t}(k)\right|^{2} \\
& =\int d k \int p_{t}\left(t_{1}\right) e^{i c k t_{1}} d t_{1} \int p_{t}\left(t_{2}\right) e^{i c k t_{2}} d t_{2} \\
& =2 \pi \int p_{t}\left(t_{1}\right) p_{t}\left(t_{2}\right)\left(\int \frac{e^{i c k\left(t_{1}-t_{2}\right)}}{2 \pi} d k\right) d t_{1} d t_{2} \\
& =(2 \pi / c) \int p_{t}\left(t_{1}\right) p_{t}\left(t_{2}\right) \delta\left(t_{1}-t_{2}\right) d t_{1} d t_{2} \\
& =(2 \pi / c) \int p_{t}^{2}(t) d t .
\end{aligned}
$$

\section{APPENDIX F: ESTIMATION OF NUMBER OF COHERENT MODES}

An order of magnitude estimate of the number of coherent modes $M$ can be made as the ratio of the radiation phase space volume $\Omega$ and the coherent phase space volume $\Omega_{R}[2,7,25,45]$ :

$$
M=\frac{\Omega}{\Omega_{R}},
$$

where

$$
\Omega_{R}=1 /(2 k)^{3} .
$$

Given that Eq. (1) is satisfied, the following expression for $\Omega$ can be used [[7] pp. 26-28],

$$
\Omega=\sqrt{\sigma_{x}^{2}+\sigma_{r}^{2}} \sqrt{\sigma_{y}^{2}+\sigma_{r}^{2}} \sigma_{r^{\prime}}^{2} c \sigma_{t} \sigma_{\omega} / \omega,
$$

where $\sigma_{\omega} / \omega$ describes the width of the radiation spectrum, $\sigma_{r}$ is the effective radiation source size, $\sigma_{r^{\prime}}$ is the angular divergence of the radiation.

For the fundamental of undulator radiation, see [[25] pp. 56, 75],

$$
\sigma_{r}=\frac{1}{4 \pi} \sqrt{\lambda_{1} L_{u}}, \quad \sigma_{r^{\prime}}=\sqrt{\frac{\lambda_{1}}{L_{u}}},
$$




$$
\sigma_{\omega} / \omega=\frac{1}{N_{u}}, \quad k=k_{1}=\frac{2 \pi}{\lambda_{1}},
$$

where $N_{u}$ is the number of undulator periods, $L_{u}$ is the length of the undulator, and $\lambda_{1}$ is the wavelength of the fundamental.

In IOTA, $1 /\left(2 k_{1}\right)=89 \mathrm{~nm}, \sigma_{r}=64 \mu \mathrm{m}, \sigma_{r^{\prime}}=1.4 \mathrm{mrad}$, $\sigma_{\omega} / \omega=1 / N_{u}=0.095$. With these values, Eq. (F1) gives $M=8.6 \times 10^{6}$.

In the Brookhaven experiment, at lattice configuration A, $1 /\left(2 k_{1}\right)=42 \mathrm{~nm}, \sigma_{r}=87 \mu \mathrm{m}, \sigma_{r^{\prime}}=0.49 \mathrm{mrad}$. The width of the radiation spectrum was determined by the monochromator, $\sigma_{\omega} / \omega=3.2 \mathrm{~nm} / 2.355 / 532 \mathrm{~nm}=2.55 \times 10^{-3}$, see Table I. With these values, Eq. (F1) gives $M=1.6 \times 10^{5}$.

[1] M. C. Teich, T. Tanabe, T. C. Marshall, and J. Galayda, Statistical Properties of Wiggler and Bending-Magnet Radiation from the Brookhaven Vacuum-Ultraviolet Electron Storage Ring, Phys. Rev. Lett. 65, 3393 (1990).

[2] F. Sannibale, G. Stupakov, M. Zolotorev, D. Filippetto, and L. Jägerhofer, Absolute bunch length measurements by incoherent radiation fluctuation analysis, Phys. Rev. Accel. Beams 12, 032801 (2009).

[3] P. Catravas, W. Leemans, J. Wurtele, M. Zolotorev, M. Babzien, I. Ben-Zvi, Z. Segalov, X.-J. Wang, and V. Yakimenko, Measurement of Electron-Beam Bunch Length and Emittance Using Shot-Noise-Driven Fluctuations in Incoherent Radiation, Phys. Rev. Lett. 82, 5261 (1999).

[4] V. Sajaev, Measurement of bunch length using spectral analysis of incoherent radiation fluctuations, in AIP Conf. Proc. 732, 73 (2004).

[5] V. Sajaev, Determination of longitudinal bunch profile using spectral fluctuations of incoherent radiation, Argonne National Laboratory Report No. ANL/ASD/ CP-100935, 2000.

[6] K.-J. Kim, Start-up noise in 3-D self-amplified spontaneous emission, Nucl. Instrum. Methods Phys. Res., Sect. A 393, 167 (1997).

[7] K.-J. Kim, Z. Huang, and R. Lindberg, Synchrotron Radiation and Free-Electron Lasers (Cambridge University Press, Cambridge, England, 2017).

[8] S. Benson and J. M. Madey, Shot and quantum noise in free electron lasers, Nucl. Instrum. Methods Phys. Res., Sect. A 237, 55 (1985).

[9] E. L. Saldin, E. Schneidmiller, and M. V. Yurkov, The physics of free electron lasers (Springer Science \& Business Media, 2013).

[10] C. Pellegrini, A. Marinelli, and S. Reiche, The physics of x-ray free-electron lasers, Rev. Mod. Phys. 88, 015006 (2016).

[11] W. Becker and M. S. Zubairy, Photon statistics of a freeelectron laser, Phys. Rev. A 25, 2200 (1982).

[12] J. W. Goodman, Statistical optics (John Wiley \& Sons, 2015).
[13] R. J. Glauber, The quantum theory of optical coherence, Phys. Rev. 130, 2529 (1963).

[14] R. J. Glauber, Coherent and incoherent states of the radiation field, Phys. Rev. 131, 2766 (1963).

[15] R. J. Glauber, Some notes on multiple-boson processes, Phys. Rev. 84, 395 (1951).

[16] S. Antipov, D. Broemmelsiek, D. Bruhwiler, D. Edstrom, E. Harms, V. Lebedev, J. Leibfritz, S. Nagaitsev, C.-S. Park, H. Piekarz et al., IOTA (Integrable Optics Test Accelerator): facility and experimental beam physics program, J. Instrum. 12, T03002 (2017).

[17] I. Lobach, K.-J. Kim, T. Shaftan, V. Lebedev, S. Nagaitsev, A. Romanov, G. Stancari, A. Valishev, A. Murokh, A. Halavanau, Z. Huang, and V. Yakimenko, Study of fluctuations in undulator radiation in the IOTA ring at Fermilab, in Proc. 10th International Particle Accelerator Conference (IPAC'19), Melbourne, Australia, 19-24 May 2019 (JACoW, Geneva, 2019) pp. 777-780.

[18] I. Lobach, V. Lebedev, S. Nagaitsev, A. Romanov, G. Stancari, A. Valishev, A. Halavanau, Z. Huang, and K.-J. Kim, Statistical properties of undulator radiation in the IOTA storage ring, arXiv:1912.06737.

[19] K.-J. Kim, Characteristics of synchrotron radiation, AIP Conf. Proc. 184, 565 (1989).

[20] C. Silberhorn, Detecting quantum light, Contemp. Phys. 48, 143 (2007).

[21] A. Meda, E. Losero, N. Samantaray, F. Scafirimuto, S. Pradyumna, A. Avella, I. Ruo-Berchera, and M. Genovese, Photon-number correlation for quantum enhanced imaging and sensing, J. Opt. 19, 094002 (2017).

[22] L. Davidovich, Sub-Poissonian processes in quantum optics, Rev. Mod. Phys. 68, 127 (1996).

[23] K.-J. Kim, Temporal and transverse coherence of selfamplified spontaneous emission, AIP Conf. Proc. 413, 3 (1997).

[24] J. Haïssinski, Exact longitudinal equilibrium distribution of stored electrons in the presence of self-fields, Il Nuovo Cimento Soc. Ital. Fis. (1971-1996) 18B, 72 (1973).

[25] J. A. Clarke, The Science and Technology of Undulators and Wigglers (Oxford University Press on Demand, New York, 2004), p. 4.

[26] I. Lobach, The source code for calculation of spectralangular distribution of wiggler radiation, https://github .com/IharLobach/wigrad (2020).

[27] I. Lobach, The source code for calculation of fluctuations in wiggler radiation, https://github.com/IharLobach/fur (2020).

[28] E. L. Saldin, E. A. Schneidmiller, and M. Yurkov, Statistical properties of radiation from VUV and $\mathrm{x}$-ray free electron laser, Opt. Commun. 148, 383 (1998).

[29] S. O. Rice, Mathematical analysis of random noise, Bell Syst. Tech. J. 23, 282 (1944).

[30] Z. Huang and K.-J. Kim, Review of x-ray free-electron laser theory, Phys. Rev. Accel. Beams 10, 034801 (2007).

[31] A. Amir, I. Boscolo, and L. R. Elias, Spontaneous emission in the waveguide free-electron laser, Phys. Rev. A 32, 2864 (1985).

[32] G. Geloni, V. Kocharyan, and E. Saldin, On quantum effects in spontaneous emission by a relativistic electron beam in an undulator, arXiv:1202.0691. 
[33] W. Becker and J. McIver, Photon statistics of the free-electron-laser startup, Phys. Rev. A 28, 1838 (1983).

[34] W. Becker and J. McIver, Fully quantized many-particle theory of a free-electron laser, Phys. Rev. A 27, 1030 (1983).

[35] A. T. Georges, From noise to coherence in a free-electron laser oscillator, in Free-Electron Generators of Coherent Radiation, edited by C. A. Brau, S. F. Jacobs, and M. O. Scully, International Society for Optics and Photonics (SPIE, Bellingham, WA, 1984), Vol. 0453, pp. 297-305.

[36] J. Gea-Banacloche, Quantum theory of the free-electron laser: Large gain, saturation, and photon statistics, Phys. Rev. A 31, 1607 (1985).

[37] S. Gottschalk, R. Kelly, M. Offenbacker, and J. Zumdieck, Design and performance of the NLCTA-Echo 7 undulators, in Proc. 34th International Free-Electron Laser Conference, Nara, Japan, 26-31 Aug. 2012.

[38] V. Lebedev, Understanding lifetimes, beam sizes and scattering in IOTA, FAST/IOTA Collaboration Meeting, Fermilab, Jun 15-17, 2020.

[39] Specifications of the InGaAs PIN photodiode G11193-10R at the Hamamatsu website, https://www.hamamatsu.com/
us/en/product/type/G11193-10R/index.html, accessed: 2019-09-10.

[40] O. Chubar, A. Fluerasu, L. Berman, K. Kaznatcheev, and L. Wiegart, Wavefront propagation simulations for beamlines and experiments with "Synchrotron Radiation Workshop", in J. Phys. Conf. Ser., Vol. 425 (IOP Publishing, London, 2013) p. 162001.

[41] S. Nagaitsev, Intrabeam scattering formulas for fast numerical evaluation, Phys. Rev. Accel. Beams 8, 064403 (2005).

[42] K. L. Bane, H. Hayano, K. Kubo, T. Naito, T. Okugi, and J. Urakawa, Intrabeam scattering analysis of measurements at KEK's accelerator test facility damping ring, Phys. Rev. Accel. Beams 5, 084403 (2002).

[43] A. W. Chao, K. H. Mess et al., Handbook of Accelerator Physics and Engineering (World Scientific, Singapore, 2013), Chap. 2, pp. 156-159.

[44] J. Hubbard, Calculation of Partition Functions, Phys. Rev. Lett. 3, 77 (1959).

[45] K.-J. Kim, Analysis of optical stochastic cooling including transverse effects, in Proc. 16th Particle Accelerator Conference, Dallas, Texas, 1-5 May 1995, Vol. 4 (IEEE, New York, 1995) pp. 2786-2788. 